\title{
Design of Chalcones of 7-Azaindole as Raf-B Inhibitors
}

\author{
D. Giles*, V.N. Saiprabha, G. Yeshna \\ Acharya and BM Reddy College of Pharmacy, Achit Nagar Post, Bengaluru - 560 107, Karnataka, India.
}

\section{ARTICLE DETAILS}

\section{Article history:}

Received as Revised 05 November 2018

Accepted 23 November 2018

Available online 07 January 2019

\section{Keywords:}

7-Azaindole

Anticancer

Chalcone

\begin{abstract}
A B S T R A C T
The present work deals with the design of 7-azaindole derivatives for its Raf-B inhibition. All the designed compounds follows Lipinski's rule of five. In silico ADME predictions of all the designed compounds suggests that none of the compounds have problem with bioavailability. The compounds were designed on the binding affinity towards the Raf-B inhibition. It was observed that few of the designed compounds were found to have significant interaction with the active site of the receptor. The compounds possessing 3-hydroxyl-2-methyl as substitution in chalcone was found to possess maximum docking score than other designed compounds.
\end{abstract}

\section{Introduction}

The Raf family kinases consist of three isoforms: A-Raf, B-Raf and C-Raf. B-Raf represents an interesting target for anticancer drug development as it is mainly involved in the Ras-Raf-MEK-ERK signal transduction pathway participating in cell proliferation and cell survival [1, 2]. B-Raf kinase is found to frequently mutate in melanomas, with the Val600/ Glu600 (V600E) transition representing the most common mutation [3], accounting for over $90 \%$ in all of the B-Raf oncogenic mutants reported to date. Furthermore, this mutation type causes B-Raf to signal independently from upstream regulation [4]. The clinical approval of Vemurafenib and Dabrafenib for the treatment of metastatic melanoma bearing the B-RafV600E mutation has further confirmed that B-RafV600E may be an attractive target for anticancer therapy [5].

Molecular docking is used routinely to predict the binding strength of ligands in the target [6]. The efficacy of molecular docking may be significantly enhanced through discriminatory analysis for the selection of docking scores [7]

7 -azindole derivatives have been found to be an important scaffold in the anticancer drug like Vemurafenib [8]. Chalcone derivatives have been an important class of compounds which is used as anticancer agents [9]. Hence conjugating the azaindole ring with chalcone could improve the binding affinity of compound which in turn the pharmacological activity increases.

As part of our continued interest in drug design of Raf-B inhibitors as anticancer agents, the present strategy, involves designing for chalcone derivatives of 7-azaindole as Raf-B inhibitors by docking study.

\section{Experimental Methods}

\subsection{Drug-Likeness Evaluation}

Drug-likeness rules are used for fast calculation of druglike properties of a molecule. The predicted drug-likeness properties [10] like molecular weight, AlogP, Hydrogen bond donars, Hydrogen bond acceptors and number of rotatable bonds were reported in Table 1.

\subsection{ADME Studies}

All the designed compounds were subjected to absorption, distribution, metabolism and excretion descriptors using Discovery Studio 3.5. This study provides the insight into the pharmacokinetic property of the designed compounds [11]. The results were depicted in Fig. 1.

Table 1 Drug-likeness evaluation of the designed compounds

\begin{tabular}{|c|c|c|c|c|c|}
\hline Ligand & $\begin{array}{l}\text { No of H- } \\
\text { bond } \\
\text { donor }\end{array}$ & $\begin{array}{l}\text { No of H- } \\
\text { bond } \\
\text { acceptor }\end{array}$ & $\begin{array}{l}\text { Molecular } \\
\text { weight } \\
(\mathrm{g} / \mathrm{mol})\end{array}$ & $\log P$ & $\begin{array}{l}\text { No of } \\
\text { rotatable } \\
\text { bonds }\end{array}$ \\
\hline 1. & 2 & 4 & 298.724 & 3.466 & 3 \\
\hline 2. & 4 & 5 & 279.293 & 2.055 & 3 \\
\hline 3. & 4 & 5 & 279.293 & 2.055 & 3 \\
\hline 4. & 2 & 4 & 278.305 & 3.288 & 3 \\
\hline 5. & 2 & 4 & 278.305 & 3.288 & 3 \\
\hline 6. & 1 & 4 & 357.201 & 3.776 & 4 \\
\hline 7. & 1 & 4 & 357.201 & 3.776 & 4 \\
\hline 8. & 1 & 5 & 308.331 & 3.011 & 5 \\
\hline 9. & 2 & 4 & 282.269 & 3.007 & 3 \\
\hline 10. & 1 & 3 & 248.279 & 3.043 & 3 \\
\hline 11. & 1 & 4 & 312.75 & 3.692 & 4 \\
\hline 12. & 1 & 4 & 312.75 & 3.692 & 4 \\
\hline
\end{tabular}

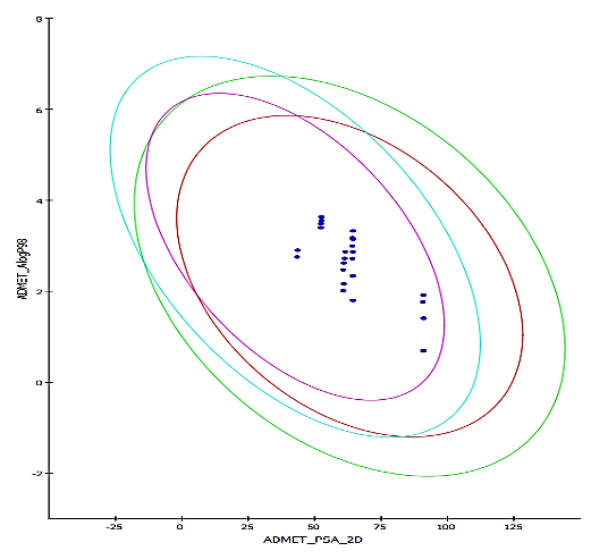

Fig. 1 ADME investigation of the designed compounds

\subsection{Docking}

\subsubsection{Preparation of the Target Protein}

The crystal structure of Raf-B (pdb: 4EHG) active site was employed as the template for molecule docking [12]. All crystallographic water molecules were removed. Hydrogen atoms were added using CHARMm 
force field. The site for docking was selected from the receptor cavity and protein was prepared using protein preparation tool.

\subsubsection{Ligand Preparation}

Aza indole based ligand library was prepared and minimized using CHARMm force field [13]. The ligands were designed on the basis of binding group of azaindole to fit into the 1CX2 effectively. Methyl, amino, hydroxy, chloro, bromo, iodo, fluro, and nitro groups in phenyl ring were considered as mono or substituent form in chalcone. These compounds were designed on the basis of the binding affinity and the target specificity. A total of 12 compounds were designed for the docking study. All conformers were treated with the ligand minimization of the receptor ligand interaction module. Minimized ligand and protein were used for the docking studies.

\subsubsection{Docking Studies}

To identify the molecule binding interaction of the designed compound with the target, all the compounds were docked into the active sites of both the targets. Computational studies of novel azaindole derivatives where carried out using Discovery Studio 3.5. CDOCKER interaction energy, hydrogen bonding and the interacting amino acid involved in the binding where used to predict the effect of binding with the target. The results of docking were listed in Table 2. Docking poses were mentioned in Figs. 2 and 3 .

Table 3 Docking study of Raf-B inhibitors

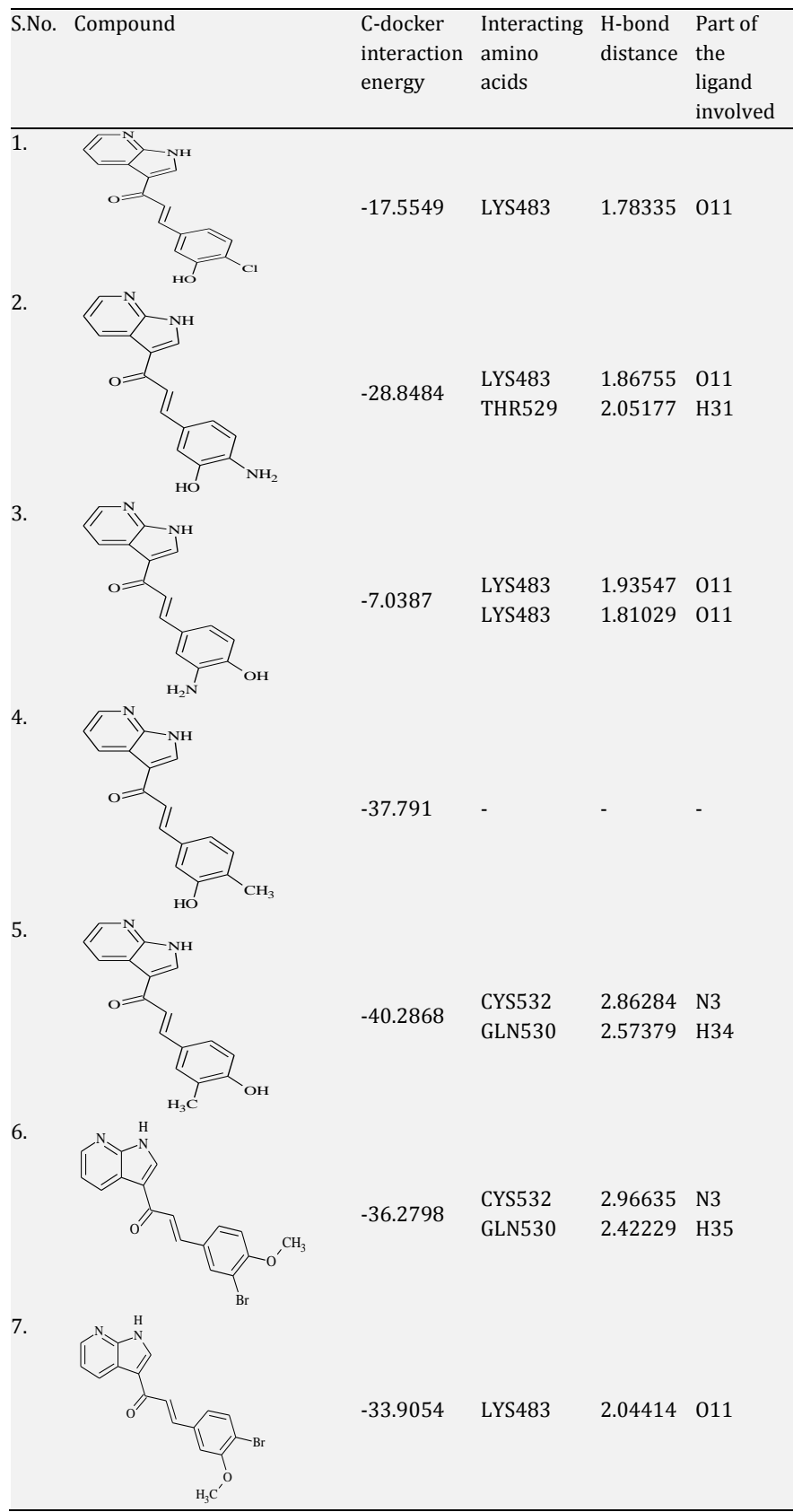

https://doi.org/10.30799/jacs.183.18040404
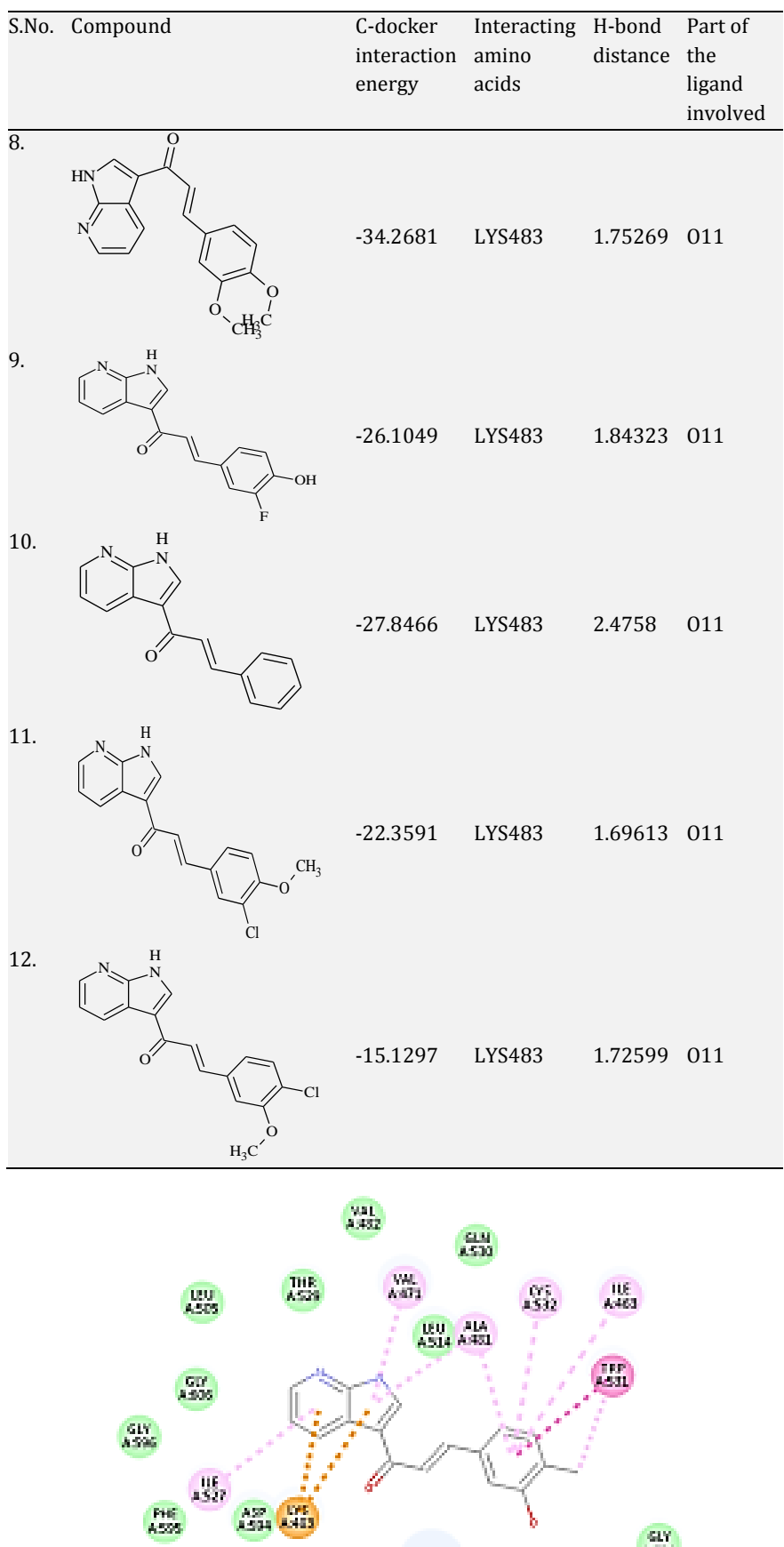

648

$\sin _{x \rightarrow 5}$

Fig. 2 Binding of compound 4 with Raf B
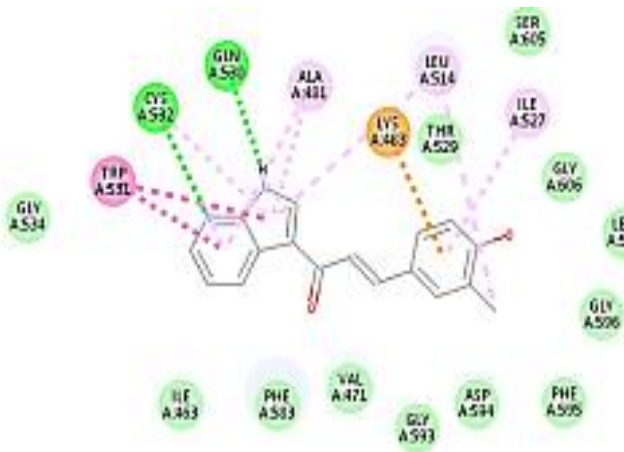

Fig. 3 Binding of compound 3 with Raf B

\section{Results and Discussion}

In this research work, we have designed 12 compounds for its possible Raf-B inhibition. All the minimized ligands were carried out for their 
ADME studies. All the compounds were well in accordance to the ADME parameters. It suggest that the compounds does not have any problem with the ADME. All the designed compounds also follow Lipinski Rule 5. Docking study was carried out for the designed compounds. It's effect of substitution in 3,4-substitution in phenyl ring when compared to that of the non-substituent.

Amino, chloro, hydroxy, methyl, methoxy, bromo, fluro were the substituent's studied in this research work. It was observed that presence of methyl and hydroxyl group as substituent at 3,4-position possess good CDOCKER interaction energy of -40.2868 toward the Raf-B target when compared to that of other substituents with two hydrogen bonding with the target. Reduction in the CDOCKER interaction energy of -37.791was found while interchanging the position. The compounds does not form hydrogen bonds with the target. CDOCKER interaction energy of unsubstituted phenyl group in the chalcone was found to be -27.8466 with one hydrogen bond of distance 2.4758. CDOCKER interaction energy of bromo and methoxy group as substituent in 3,4- position was found to be -36.2798 and -33.9054 respectively. The 3-bromo-4-methoxy group as substitutent was found to possess high interaction energy and two hydrogen bonds when compared to that of 4-bormo-3-methoxy group. 3methoxy-4-chloro as substituent in phenyl ring of chalcone possess less CDOCKER interaction energy when compare to that of 3-chloro-4-methoxy group. Both interact with same amino acid LYS483 with one hydrogen bond each. Presence of dimethoxy group as substituent in phenyl ring possess good CDOCKER interaction energy of -34.2681. It forms one hydrogen bond with LYS483 with a bond length of 1.75269. High CDOCKER interaction energy difference was found with for compound with chloro and amino group as substitutent at $3,4^{\text {th }}$ position in phenyl ring. Presence of 3-hydroxy-4-amino group as substituent possess good CDOCKER interaction energy of -28.8484 with two hydrogen bonds while the interaction reduces to -7.0387 on change of position of as 3-amino-4hydroxyl group. Moderate CDOCKER interaction energy of -17.5549 was observed for compound with 3-hyroxy-4-chloro as substitution. Presence of 3-fluro-4-hydroxy group possesses good CDOCKER interaction energy of -26.1049 with one hydrogen bond with LYS483.

\section{Conclusion}

Twelve chalcone derivatives of 7-azaindole were designed for its Raf-B inhibition. The effect of substitution at 3,4-position was studied. It was found that all the designed compounds follow Lipinski's rule 5 and does not have any problem with ADME. Docking study reveals that all the compounds docked effectively towards the Raf-B target. Among all the designed compounds, substitution at 3-methyl-4-hydroxy group as substitution possesses good CDOCKER interaction energy when compared to that of other compounds. 4-methyl-3-hydroxyl group also possess good interaction energy. All the reports suggests that the presence of methyl and hydroxyl group as substitution at 4 and 3-position increases binding with Raf-B.

\section{Acknowledgement}

Authors are thankful to Rajiv Gandhi University of Health Science Karnataka for the financial support. The authors are also thankful to the Chairman and the Principal of Acharya \& BM Reddy College of Pharmacy, Bangalore for providing facilities to carry out the work successfully.

\section{References}

[1] D.T. Leicht, V. Balan, A. Kaplun, V. Singh-Gupta, L. Kaplun, M. Dobson, et al., Raf Kinases: Function, regulation and role in human cancer, Biochimica. Biophysica. Acta. 1773 (2007) 1196-1212.

[2] I. Hmitou, S. Druillennec, A. Valluet, C. Peyssonnaux, A. Eychene, Differentia regulation of B-Raf isoforms by phosphorylation and autoinhibitory mechanisms, Mol. Cell Biol. 27 (2007) 31-43.

[3] D. Zippel, G. Markel, R. Shapira-Frommer, G. Ben-Betzalel, D. Goitein, E. BenAmi, et al., Perioperative BRAF inhibitors in locally advanced stage III melanoma, J. Surg. Oncol. 116 (2017) 856-861.

[4] A.M. Acosta, S.S. Kadkol, Mitogen-activated protein kinase signaling pathway in cutaneous melanoma: An updated review, Arch. Pathol. Lab. Med. 140 (2016) 1290-1296.

[5] E. Cardoso, T. Mercier , A.D. Wagner, K. Homicsko, O. Michielin, K. EllefsenLavoie, et al., Quantification of the next-generation oral anti-tumor drugs dabrafenib, trametinib, vemurafenib, cobimetinib, pazopanib, regorafenib and two metabolites in human plasma by liquid chromatography-tandem mass spectrometry, J. Chromatogr. B. 1083 (2018) 124-136.

[6] P.F. Wang, Y.J. Zhang, D. Wang, H.M. Hu, Z.C. Wang, C. Xu , et al., Design, synthesis, and biological evaluation of new B-Raf(V600E) kinase inhibitors, Bioorg. Med. Chem. 26(9) (2018) 2372-2380.

[7] S.A. Abdelatef, M.T. El-Saadi, N.H. Amin, A.H. Abdelazeem, H.A. Omar, K.R.A Abdellatif, Design, synthesis and anticancer evaluation of novel spirobenzo $[\mathrm{h}]$ chromene and spirochromane derivatives with dual EGFR and BRAF inhibitory activities, Eur. J. Med. Chem. 150 (2018) 567-578.

[8] S. Sun, Z. He, M. Huang, N. Wang, Z. He, X. Kong, et al., Design and discovery of thioether and nicotinamide containing sorafenib analogues as multikinase inhibitors targeting B-Raf, B-Raf(V600E) and VEGFR-2, Bioorg. Med. Chem. 26(9) (2018) 2381-2391.

[9] G. Wang, Z. Peng, J. Zhang, J. Qiu, Z. Xie, Z. Gong, Synthesis, biological evaluation and molecular docking studies of aminochalcone derivatives as potential anticancer agents by targeting tubulin colchicine binding site, Bioorg. Chem. 78 (2018) 332-340.

[10] I. Azad, M. Nasibullah, T. Khan, F. Hassan, Y. Akhter, Exploring the novel heterocyclic derivatives as lead molecules for design and development of potent anticancer agents, J. Mol. Graph. Model. 18 (2018) 211-228.

[11] M. Szumilak, W. Lewgowd, A. Stanczak, In Silico ADME studies of polyamine conjugates as potential anticancer drugs, Acta. Pol. Pharma. 73 (2016) 11911200.

[12] V. Previtali, C. Trujillo, J.C. Boisson, H. Khartabil, E. Henon, I. Rozas, Development of the first model of a phosphorylated, ATP/ $\mathrm{Mg}^{2+}$-containing BRaf monomer by molecular dynamics simulations: a tool for structure-based design, Phys. Chem. Chem. Phys. 46 (2017) 31177-31185.

[13] F.Y. Lin, P.E. Lopes, E.D. Harder, B. Roux, A.D. MacKerell, Polarizable force field for molecular ions based on the classical drude oscillator, J. Chem. Inf. Model. 58(5) (2018) 993-1004. 